\title{
NOTIZEN
}

\section{Zur Renormalisierung der Tamm-Dancoff-Gleichungen}

Von H. Lehmann

Max-Planck-Institut für Physik, Göttingen

(Z. Naturforschg. 8a, 579-580 [1953]; eingeg. am 3. August 1953)

In Arbeiten von Lévy ${ }^{1}$ ist die nichtadiabatische Methode von Tamm-Dancoff erweitert und auf das Proton-Neutron-System angewandt worden. Eine befriedigende Behandlung von Strahlungskorrekturen war dabei zunächst nicht durchführbar. Kürzlich sind von $\mathrm{Cini}^{2}$ sowie von $\mathrm{Zimmermann^{3 }}$ neue Formulierungen der nichtadiabatischen Gleichungen angegeben worden, die dem Wunsch nach expliziter Kovarianz der verwendeten Ausdrücke Rechnung tragen. Cini hat dabei die Vermutung ausgesprochen, daß es nunmehr möglich sei, durch Renormalisierung von Masse und Kopplungskonstante alle bei den Korrekturtermen zunächst auftretenden divergenten Glieder zu eliminieren.

Zweck dieser Note ist zu zeigen, daß bei Anwendung der Tamm-Dancoff-Methode divergente Ausdrücke vorkommen, die sich nicht (wie bei der $S$-Matrix) als Renormalisierungsterme interpretieren lassen und einer konsequenten Durchführung dieser Methode auch bei weitgehend invarianter Formulierung im Wege stehen.

Wir beziehen uns im folgenden auf das Deuteronproblem. Zur Beschreibung dieses Systems wird eine Wahrscheinlichkeitsamplitude $\chi_{t}\left(x^{\prime}, x^{\prime \prime}\right)=\left[\Phi_{0}, \psi^{(+)}\right.$ $\left.\left(x^{\prime}\right) \psi^{(+)}\left(x^{\prime \prime}\right) \Phi(t)\right]$ benutzt. Zustandsvektoren $\Phi$ und Spinoroperatoren $\psi$ sind Größen der Wechselwirkungsdarstellung; $\Phi_{0}$ ist das ,,freie" Vakuum und ( + ) bedeutet den positiven Frequenzanteil. Die Funktion $\chi_{t}\left(x^{\prime}\right.$, $\left.x^{\prime \prime}\right)$ genügt einer homogenen Integralgleichung

$$
\chi=K \chi \text {. }
$$

Der Integralkern $K$ ist als Potenzreihe in der Kopplungskonstante $G^{2}$ definiert; die einzelnen Beiträge zu $K$ kann man durch die $S$-Matrixgraphen mit zwei einlaufenden und zwei auslaufenden Nukleonlinien charakterisieren. Für die inneren Linien eines Graphen sind $S_{\mathrm{F}}$-bzw. $\Delta_{\mathrm{F}}$-Funktionen einzusetzen. Während jedoch bei der $S$-Matrix über alle Zeiten integriert wird, erstreckt sich hier das Integrationsgebiet nur bis zu endlichen Zeiten.

Da dieselben invarianten Funktionen wie bei der $S$-Matrix auftreten, liegt es nahe ${ }^{2}$, die Behandlung von Selbstenergie- und Vertexteilen mit den von der $S$-Matrix her bekannten Methoden aufzugreifen. D.h. man zerlegt die betreffenden Ausdrücke in divergente und konvergente Bestandteile und zeigt, daß die ersteren als Renormalisierungsterme anzusprechen sind. Im Gegensatz zur $S$-Matrix ist hier jedoch - wegen der endlichen Zeiten - noch eine Integration über den konvergenten Teil auszuführen, die erneut zu Divergenzen Anla $\beta$ geben kann (und wird, s.u.).

Das geschilderte Verfahren führt tatsächlich bei den $G^{2}$-Korrekturen, die durch den Selbstenergieeffekt eines Nukleons entstehen, zum gewünschten Erfolg; nach Abspaltung der Renormalisierungsglieder verbleibt ein endlicher Beitrag. Wir werden jedoch zeigen, daß bei allen Graphen, die in sich geschlossene Nukleonlinien (closed loops) enthalten, divergente Terme übrigbleiben.

Dies soll an einem einfachen Feynman-Graphen dieser Art<smiles>CO[14C](C)(C)C</smiles>

demonstriert werden, der zur rechten Seite der Integralgleichung folgenden Beitrag liefert ${ }^{2}$ [unwesentliche Faktoren werden unterdrückt]:

$$
\begin{aligned}
& \begin{array}{l}
I=\int \mathrm{d}^{4} x_{1} \mathrm{~d}^{4} x_{2} \mathrm{~d}^{4} x_{3} \mathrm{~d}^{4} x_{4} S^{(+)}\left(x^{\prime}-x_{3}\right) \gamma_{5} S^{(+)}\left(x^{\prime \prime}-x_{4}\right) \gamma_{5} \Delta_{\mathrm{F}}\left(x_{3}-x_{1}\right) \Delta_{\mathrm{F}}\left(x_{2}-x_{4}\right) \pi\left(x_{1}-x_{2}\right) \\
\quad \cdot \sum_{P(\alpha, \beta, \gamma, \delta)} \theta\left(t-t_{\alpha}\right) \theta\left(t_{\alpha}-t_{\beta}\right) \theta\left(t_{\beta}-t_{\gamma}\right) \theta\left(t_{\gamma}-t_{\delta}\right) \chi_{t} \delta\left(x_{3}, x_{4}\right) .
\end{array} \\
& \text { Hierbei ist }
\end{aligned}
$$

$$
\begin{gathered}
\pi\left(x_{1}-x_{2}\right)=S p\left\{S_{\mathrm{F}}\left(x_{2}-x_{1}\right) \gamma_{5} S_{\mathrm{F}}\left(x_{1}-x_{2}\right) \gamma_{5}\right\} ; \\
\theta(x)=\left\{\begin{array}{l}
1 ; x>0 \\
0 ; x<0
\end{array} .\right.
\end{gathered}
$$

Die Argumente $t_{\alpha} \ldots t_{\delta}$ der $\theta$-Funktionen durchlaufen alle Permutationen der Zeiten $t_{1} \ldots t_{4}$.

Für die Fourier-Transformierte erhält man:

$$
\begin{aligned}
\tilde{I}=\int \frac{\mathrm{d}^{3} k}{E_{\bar{K}}^{2}} \tilde{\chi}\left(\mathfrak{p}_{1}-\mathfrak{t}, \mathfrak{n}_{1}+\mathfrak{l}\right) \int \mathrm{d}^{4} q \delta^{(3)}(\mathfrak{f}-\mathfrak{q}) \\
\cdot \pi(q) f\left(E_{K}, q_{0}\right) ;\left(E_{\bar{K}}^{2}=\mathfrak{l}^{2}+\mu^{2}\right) .
\end{aligned}
$$

${ }^{1}$ M. M. Lévy, Physic. Rev. 88, 72, 725 [1952].

2 M. Cini, Nuovo Cimento 10, 526, 614 [1952].
Hier ist $\tilde{\chi}$ im wesentlichsten die Fourier-Transformierte von $\chi$.

$f\left(E_{K}, q_{0}\right)$ ist eine rationale Funktion, die sich durch Ausführung der Zeitintegration ergibt. Wir verzichten auf die explizite Angabe des etwas umständlichen Ausdrucks, sondern bemerken, daß $f \sim 1 / q_{0}{ }^{2}$ für große Werte von $q_{0}$ ( $f$ enthält außerdem Glieder $\sim 1 / q_{0}$, die jedoch zur $q$-Integration nicht beitragen).

Wir müssen nun das Integral über $q$ betrachten, das die Korrekturterme enthält. In der Funktion $f$ kom-

${ }^{3}$ W. Zimmermann, Z. Physik, erscheint demnächst. 
men dabei die endlichen Zeiten zur Auswirkung [für das entsprechende $S$-Matrixelement gilt $f \sim \delta\left(E_{K}-q_{0}\right)$ und die $q$-Integration liefert einfach $\pi(k)]$. Die erwähnte Aufspaltung von $\pi$ in divergente und konvergente Terme lautet ${ }^{2}$

$$
\pi(q)=A+C\left(q_{\bar{\lambda}}^{2}+\mu^{2}\right)+\pi^{c}(q) ;
$$

$A$ und $C$ sind divergente Konstanten. Für große Werte von $q_{\dot{\lambda}}^{2}$ gilt $\pi^{c}(q) \sim q_{\bar{\lambda}}^{2}$.

Man sieht jetzt unmittelbar, daß die hier notwendige Integration über die, ,konvergente" Funktion $\pi^{c}$ zu Divergenzen Anlaß gibt, die sich nicht als Renormalisierungsglieder deuten lassen. Weiterhin stößt die Interpretation von $C$ als Renormalisierungskonstante wegen der divergenten $q$-Integration auf Schwierigkeiten. - Dieselben Verhältnisse liegen bei allen Graphen vor, die Mesonselbstenergieteile enthalten.

Die diskutierten Schwierigkeiten bei der Auswertung von Strahlungskorrekturen treten ebenfalls auf, wenn man versucht, die Matrixelemente des Transfor-

${ }^{4}$ E.C.G. Stueckelberg, Physic. Rev. 81, 130 [1951].

\section{Zur Kernisomerie beim ${ }^{192} \mathrm{Ir}$}

Von G. Weber und A. Flammersfeld

Aus dem Max-Planck-Institut für Chemie, Mainz

(Z. Naturforschg. 8a, 580-581 [1953]; eingeg. am 28. Juli 1953)

Bei der Bestrahlung von Ir mit langsamen Neutronen tritt neben anderen eine starke Aktivität von $T=$ 1,45 min Halbwertszeit auf, die ${ }^{192} \mathrm{Irm}^{\mathrm{m}}$ darstellt und zum ${ }^{192} \operatorname{Ir}(T=74,3 \mathrm{~d})$ isomer ist $^{1}$. Das $1,45 \mathrm{~min}$ Isomer wurde im folgenden genauer untersucht, weil außer gewöhnlichem Isomeren-Übergang auch noch Übergang durch 2-Quantenzerfall (mit kontinuierlicher Verteilung der $\gamma$-Energie) behauptet worden ist ${ }^{2}$.

Die Anregungsenergie des isomeren Zustandes ist von $\mathrm{Hole}^{3}$ und Cald well ${ }^{4}$ aus den Energien der Konversionselektronen zu $55,5 \mathrm{KeV}$ bzw. $57,4 \mathrm{KeV}$ gemessen worden. Außer diesen Elektronenlinien, der unkonvertierten $\gamma$-Strahlung und der charakteristischen Röntgen-(L-)Strahlung wurde von Goldhaber und Mitarbb. ${ }^{2}$ noch die Existenz eines $\gamma$-Kontinuums mit der mittleren Energie $\mathrm{E} \approx 30 \mathrm{KeV}$ und der oberen Grenze $\approx 60 \mathrm{KeV}$ behauptet. Zur Erklärung dieses Kontinuums wurde der von Sachs ${ }^{5}$ diskutierte Zerfallsmechanismus herangezogen. Es war nicht angegeben, ob das $\gamma$-Kontinuum teilweise konvertiert sein soll oder nicht. Wir haben zunächst versucht, eventuelle Umwandlungselektronen des Kontinuums mit Hilfe eines Slätis-Siegbahnschen $\beta$-Spektrometers nachzuweisen.

${ }^{1}$ E. M. Mc Millan, N. Kamen u. S. Ruben, Physic. Rev. 52, 375 [1937]; V. Fomin u. F. G. Houtermans, Physik. Z. Sowjetunion 9, 273 [1936]; L. Seren, H.N. Friedlander u. S.H.Turkel, Physic. Rev. 72, 888 [1947].

${ }^{2}$ M. Goldhaber, C.O, Muehlhouse u. S. H. Tur- mationsoperators $U(t,-\infty)$ zu berechnen ${ }^{4}$. Der Ursprung der (gegenüber der $S$-Matrix) neu auftretenden divergenten Terme ist in der scharfen zeitlichen Begrenzung der Integrationsgebiete zu suchen. Es ist bekannt $^{5}$, daß auch die Erwartungswerte von Feldoperatoren (z. B. des Stromes in der Quantenelektrodynamik in einem Raum-Zeitgebiet unendlich groß werden, sofern dieses Gebiet zeitlich scharf begrenzt wird.

Man erhält endliche Resultate, wenn man die scharfe Begrenzung des Integrationsbereiches aufhebt; d.h. in unserem Fall die Sprungfunktion $\theta$ durch eine stetige Funktion ersetzt. Jedoch ist unseres Wissens bisher keine konsequente Begründung einer derartigen Abänderung vorgenommen worden.

Wir glauben deshalb, daß es zu einer konsistenten Behandlung von gebundenen Zuständen notwendig ist, statt der Amplitude $\chi_{t}\left(x^{\prime}, x^{\prime \prime}\right)$ die von Bethe und Salpeter eingeführte ,Wellenfunktion“ zu benutzen. Die Unterschiede zwischen beiden Methoden werden in der erwähnten Arbeit von Zimmermann ${ }^{3}$ diskutiert.

${ }^{5}$ Vgl. z. B. E. Corinaldesi, Nuovo Cimento 9, 194 [1952].

Das Fenster des Geiger-Müller-Zählrohres war mit einer $0,08 \mathrm{mg} / \mathrm{cm}^{2}$ dicken Zaponlackfolie verschlossen, so daß noch Elektronen von $8 \mathrm{KeV}$ meßbar waren. Die Präparate bestanden aus besonders dünnen Iridiumschichten auf dünnen Unterlagen; die Aktivierung erfolgte durch Bestrahlung mit langsamen Neutronen am hiesigen Neutronen-Generator. Das Ergebnis zeigt Abb. $1 \mathrm{~A}$. Die intensiven Linien rühren von dem Isomeren-Übergang mit Umwandlung in der L- und MSchale her. Die $\gamma$-Energie ergibt sich daraus zu 56,0 $\mathrm{KeV}$ in guter Übereinstimmung mit den früheren Autoren. Eventuelle kontinuierliche Umwandlungselektronen sollten bei kleinen Energien bemerkbar sein. Die in Kurve I vorhandenen Elektronen kleinerer Energie sind offensichtlich auf Rückstreuung an der Präparatunterlage und der Streuung im Präparat zurückzuführen, wie ein Vergleich der Kurven I-III zeigt.

Während Kurve I mit einem Präparat von $0,3 \mathrm{mg} /$ $\mathrm{cm}^{2}$ aus Ir-Pulver auf einer $1 \mu$-Glimmerfolie erhalten ist, zeigt Kurve II mit $0,25 \mathrm{mg} / \mathrm{cm}^{2}$ Ir auf $1 \mu$-Zaponlack bereits wesentlich weniger Streuelektronen und Kurve III mit $0,15 \mathrm{mg} / \mathrm{cm}^{2}$ Iridium auf $0,2-\mu$-Zaponlack so wenig, daß kein Unterschied mehr gegenüber anderen normalen Isomerenübergängen zu bemerken ist. Aus der letzteren Kurve kann daher geschlossen werden, daß die Intensität eines etwa vorhandenen Elektronenkontinuums weniger als $3 \%$ betragen muß.

Weiter wurde nach unkonvertierter Strahlung von dem behaupteten $\gamma$-Kontinuum gesucht. Um die Messungen auf die Zahl der Isomeren- Übergänge beziehen

kel, Physic. Rev. 71,372 [1947]; E. Der Mateosian u. M. Goldhaber, Physic. Rev. 82, 115 [1951].

${ }^{3}$ N. Hole, Arkiv Mat. Astronomi Fysik 36, No. 9 [1948].

${ }^{4}$ L. R. Caldwell, Physic. Rev. 78, 407 [1950].

${ }^{5}$ R.G. Sachs, Physic. Rev. 57, 194 [1940]. 